\title{
Evaluación externa de los resultados serológicos en los bancos de sangre de Colombia
}

\author{
Mauricio Beltrán Durán ${ }^{1}$ y Maribel Ayala Guzmán ${ }^{1}$
}

RESUMEN Objetivo. Analizar los resultados serológicos obtenidos en los bancos de sangre colombianos que participan en el programa externo de calidad (PEC) con el fin de mejorar la calidad del tamizaje de la sangre según los principales marcadores serológicos de enfermedades infecciosas de posible transmisión sanguínea.

Métodos. Se evaluó un panel de seis sueros con diferente reactividad y positividad a anticuerpos contra los virus de la inmunodeficiencia humana (VIH) 1-2, el virus de la hepatitis C (VHC), Trypanosoma cruzi, Treponema pallidum y el virus linfotrópico de células $T$ humanas (HTLV), y contra el antígeno de superficie del virus de la hepatitis B (HBsAg). Las técnicas de tamizaje utilizadas fueron el ensayo de inmunoadsorción enzimática (ELISA), el inmunoensayo enzimático de micropartículas (MEIA) y la hemaglutinación (HA). Se solicitó a los bancos de sangre participantes que aplicaran a los sueros las pruebas que diariamente realizaban para el tamizaje de unidades de sangre y que enviaran los resultados a la Coordinación Nacional de Bancos de Sangre del Instituto Nacional de Salud de Colombia.

Resultados. De 46 bancos de sangre que participaron, 43 (93\%) devolvieron los resultados en el plazo indicado. La prueba de ELISA fue la más utilizada (83,02\%). Se obtuvo un total de $49(5 \%)$ resultados positivos falsos y 12 (3\%) resultados negativos falsos. De estos últimos, $50 \%$ correspondieron a la detección de la sifilis; $16,7 \%$ a la de la enfermedad de Chagas; $16,7 \%$ a la de anticuerpos anti-HBc; 8,3\% a la de anticuerpos anti-VHC y 8,3\% a la del HBsAg. Ochenta por ciento de los resultados discordantes se presentaron en 23 bancos de sangre con un volumen de menos de 6000 unidades de sangre al año, y 15\% en 5 bancos de sangre con un volumen de 6000 a 12000 unidades de sangre al año. De los bancos de sangre que recogían más de 12000 unidades anuales, solo uno notificó tres resultados positivos falsos. No se notificaron resultados negativos falsos.

Conclusiones. El porcentaje de resultados negativos falsos (3\%) obtenidos durante el PEC puede considerarse elevado, ya que las pruebas que resultan negativas durante el tamizaje de los bancos de sangre no se repiten y la decisión de declarar una unidad de sangre apta para transfusión se basa en ese único resultado. Es preciso revisar minuciosamente los procedimientos para el tamizaje de la sangre, en particular en aquellos centros que tuvieron un desempeño pobre.

Palabras clave Control de calidad, tamizaje, bancos de sangre.

1 Instituto Nacional de Salud, Bogotá, Colombia. Toda la correspondencia debe dirigirse a Mauricio Beltrán Durán a la siguiente dirección postal: Avenida El Dorado Carrera 50, Bogotá, Colombia. Fax: (57-1) 2207700 (ext. 420 ó 421). Correo electrónico: mbeltrand@hemagogus.ins.gov.co
Las pruebas de tamizaje para los marcadores de enfermedades infecciosas que pueden transmitirse por transfusión sanguínea cumplen una función muy importante, tanto en la selección de la sangre óptima para transfundir, como en el trabajo posterior con los donantes que arrojan resultados positivos durante el tamizaje. El número de pruebas utilizadas para el tamizaje 
en los bancos de sangre se ha incrementado y su calidad ha mejorado considerablemente gracias al uso de anticuerpos monoclonales, proteínas recombinantes, péptidos sintéticos $\mathrm{y}$ sistemas de detección novedosos, con lo que se han obtenido pruebas mucho más sensibles, específicas y confiables (1-3). Sin embargo, el tamizaje serológico constituye solo un componente de la cadena de estrategias con que cuentan los bancos de sangre para reducir el riesgo de transmisión de estas enfermedades infecciosas, desde la selección cuidadosa del donante mediante encuestas hasta la liberación de la sangre para su transfusión.

Los errores cometidos en cualquier etapa del procesamiento de la sangre pueden traducirse en la liberación de unidades de sangre infectada cuya transfusión podría traer consecuencias adversas para el receptor. Los bancos de sangre pueden reducir la frecuencia de errores mediante un sistema de control de calidad apoyado en las buenas prácticas para bancos de sangre $\mathrm{y}$ en medidas de control internas, las cuales deben dirigirse a evitar equivocaciones debidas a procedimientos inadecuados, a fallas en la organización de los documentos o registros, $\mathrm{u}$ a otras causas, como el uso de reactivos y equipos defectuosos $(4,5)$.

La evaluación externa del tamizaje serológico proporciona un mecanismo de autoevaluación para los bancos de sangre y es, además, un elemento importante en cualquier sistema de garantía de la calidad, ya que permite poner a prueba la eficacia de las medidas de control de calidad internas, sopesar el desempeño de los bancos de sangre y tomar medidas correctoras si se detectan deficiencias $(6,7)$.

La evaluación externa busca garantizar la eficacia de los programas para el tamizaje de la sangre, fomentar la observación de buenas prácticas de hemoterapia, y fortalecer el control de calidad interno $(8,9)$. Los programas externos de calidad (PEC) proveen, además, datos valiosos, a escala nacional y regional, sobre la frecuencia de los errores que se cometen en los bancos de sangre. Esta información puede ser útil para planear estrategias de asesora- miento, capacitación y monitoreo en los bancos e, indirectamente, medidas para evaluar la calidad de la sangre (10).

En Colombia, el Instituto Nacional de Salud (INS), por mediación del Laboratorio Nacional de Referencia y su Coordinación Nacional de Bancos de Sangre (CNBS), inició en 1996 un PEC para evaluar las prácticas de tamizaje serológico en los bancos de sangre. En este trabajo se analiza cómo se llevó a cabo este programa en los bancos que participaron en el segundo envío del año 1998.

\section{MATERIALES Y MÉTODOS}

Los sueros obtenidos a partir del plasma desfibrinado de los donantes de sangre fueron analizados en el Laboratorio Nacional de Referencia (LNR) del INS, mediante pruebas de tamizaje y pruebas confirmatorias, para determinar su reactividad. Los marcadores serológicos de infección empleados en los bancos de sangre y utilizados en esta investigación fueron los siguientes: el antígeno superficial del virus de la hepatitis B (HBsAg); anticuerpos contra virus de la inmunodeficiencia humana 1-2 (anti-VIH); contra Treponema pallidum (agente causal de la sífilis); contra Trypanosoma cruzi (agente causal de la enfermedad de Chagas); contra el antígeno nuclear del virus de la hepatitis $B$ (anti- $\mathrm{HBc}$ ); contra el virus de la hepatitis $C$ (antiVHC) y contra el virus linfotrópico de células T humanas (anti-HTLV). Los sueros fueron distribuidos en tubos de plástico con tapa de rosca en alícuotas de $2 \mathrm{~mL}$ y se enviaron por vía aérea según las normas de bioseguridad establecidas por la Asociación Internacional de Transporte Aéreo para el traslado de sustancias biológicas infecciosas y perecederas (11). Cada banco recibió un estuche de seis tubos con las siguientes características: tubo 1: positivo a anti-VIH, anti-T. pallidum y antiT. cruzi; tubo 2: negativo a todos los marcadores; tubo 3: positivo a $\mathrm{HBsAg}$, anti-T. pallidum y anti-HBc; tubo 4: positivo a anti-T. pallidum; tubo 5: positivo a anti-VHC; y tubo 6: positivo a HBsAg, anti-HBc y anti-HTLV.
Los bancos de sangre que participaron fueron seleccionados según su complejidad y el número de unidades de sangre captadas por año. De los 46 bancos escogidos, 32 eran de baja complejidad y captaban anualmente menos de 6000 unidades de sangre; 6 bancos eran de mediana complejidad y captaban entre 6000 y 12000 unidades anuales; y 5 bancos eran de mayor complejidad y recibían más de 12000 unidades de sangre al año.

Se solicitó a los participantes que incluyeran estos sueros dentro del tamizaje serológico diario y que aplicaran las pruebas que normalmente realizaban con sangre donada, sin ningún tratamiento especial. También se entregó un cuaderno estandarizado para registrar el tipo y la marca de las pruebas utilizadas, así como los resultados obtenidos y su interpretación. Los resultados enviados por los bancos fueron analizados en el INS según los códigos establecidos. Finalmente, se mandaron a cada banco los resultados correctos obtenidos por el LNR para que analizaran sus resultados.

\section{RESULTADOS}

Los sueros utilizados en este PEC se destinaron a bancos de sangre dedicados al tamizaje serológico de donantes. De los 46 bancos de sangre participantes, $43(93 \%)$ devolvieron los resultados en el plazo establecido. Aunque la mayor parte de los bancos examinaron las 6 muestras en su totalidad, un banco de sangre no realizó la prueba de la sífilis y otro omitió la del HBsAg por falta de reactivos. Las pruebas detectoras de anticuerpos anti-HBc fueron realizadas por 27 bancos de sangre y solo uno realizó la prueba para detectar anti-HTLV. Uno de los bancos realizó además pruebas de confirmación para anti-VIH, HBsAg y sífilis.

Las técnicas de tamizaje utilizadas por los bancos de sangre participantes fueron el ensayo de inmunoadsorción enzimática (ELISA), el inmunoensayo enzimatico de micropartículas (MEIA) y la hemaglutinación. Globalmente, las pruebas de tipo ELISA fueron las más utilizadas (en 83,0\% de las determina- 
CUADRO 1. Resultados serológicos de los bancos de sangre en las muestras negativas del panel

\begin{tabular}{|c|c|c|c|c|c|c|c|c|c|c|c|c|c|c|c|c|c|c|c|}
\hline \multirow[b]{3}{*}{ Marcadores } & \multicolumn{5}{|c|}{ ELISA } & \multicolumn{5}{|c|}{ MEIA } & \multicolumn{5}{|c|}{ Hemaglutinación } & \multicolumn{4}{|c|}{ Total } \\
\hline & \multirow[b]{2}{*}{$\mathrm{BS}^{\mathrm{a}}$} & \multicolumn{2}{|c|}{$\begin{array}{l}\text { Concor- } \\
\text { dancia }\end{array}$} & \multicolumn{2}{|c|}{$\begin{array}{l}\text { Positivos } \\
\text { falsos }\end{array}$} & \multirow[b]{2}{*}{$\mathrm{BS}$} & \multicolumn{2}{|c|}{$\begin{array}{l}\text { Concor- } \\
\text { dancia }\end{array}$} & \multicolumn{2}{|c|}{$\begin{array}{l}\text { Positivos } \\
\text { falsos }\end{array}$} & \multirow[b]{2}{*}{$\mathrm{BS}$} & \multicolumn{2}{|c|}{$\begin{array}{l}\text { Concor- } \\
\text { dancia }\end{array}$} & \multicolumn{2}{|c|}{$\begin{array}{l}\text { Positivos } \\
\text { falsos }\end{array}$} & \multirow[b]{2}{*}{ BS } & \multicolumn{2}{|c|}{$\begin{array}{c}\text { Concordancia } \\
\text { total }\end{array}$} & \multirow{2}{*}{$\begin{array}{c}\text { Postivos } \\
\text { falsos }\end{array}$} \\
\hline & & No. & $\%$ & No. & $\%$ & & No. & $\%$ & No. & $\%$ & & No. & $\%$ & No. & $\%$ & & No. & $\%$ & \\
\hline VIH & 40 & 199 & 99,5 & 1 & 0,5 & 2 & 10 & 100 & 0 & 0 & 1 & 0 & 0 & 5 & 100 & 43 & 209 & 97,2 & 6 \\
\hline $\mathrm{HBsAg}$ & 36 & 139 & 96,5 & 5 & 3,5 & 6 & 18 & 75,0 & 6 & 25,0 & & & & & & 42 & 157 & 93,5 & 11 \\
\hline Anti-HBc & 23 & 91 & 98,9 & 1 & 1,08 & 3 & 11 & 91,6 & 1 & 8,4 & 1 & 2 & 50,0 & 2 & 50,0 & 27 & 104 & 96,3 & 4 \\
\hline $\mathrm{VHC}$ & 34 & 165 & 97,0 & 5 & 3.0 & 7 & 31 & 88,5 & 4 & 11,5 & 1 & 9 & 90,0 & 1 & 10,0 & 42 & 205 & 95,3 & 10 \\
\hline Chagas & 42 & 168 & 100 & 0 & 0 & & & & & & 1 & 1 & 25,0 & 3 & 75,0 & 43 & 169 & 98,3 & 3 \\
\hline Sífilis & & & & & & & & & & & 42 & 153 & 91,0 & 15 & 9.0 & 42 & 153 & 91,1 & 15 \\
\hline HTLV & 1 & 5 & 100 & 0 & 0 & & & & & & & & & & & 1 & 5 & 100 & 0 \\
\hline Total & & 767 & 98,5 & 12 & 1,5 & & 70 & 86,41 & 11 & 13,5 & & 165 & 86,4 & 26 & 13,6 & & 1002 & 95,3 & 49 \\
\hline
\end{tabular}

a BS: Número de bancos de sangre que realizaron la prueba correspondiente.

CUADRO 2. Resultados serológicos de los bancos de sangre en las muestras positivas del panel

\begin{tabular}{|c|c|c|c|c|c|c|c|c|c|c|c|c|c|c|c|c|c|c|c|}
\hline \multirow[b]{3}{*}{ Marcadores } & \multicolumn{5}{|c|}{ ELISA } & \multicolumn{5}{|c|}{ MEIA } & \multicolumn{5}{|c|}{ Aglutinación } & \multicolumn{4}{|c|}{ Total } \\
\hline & \multirow[b]{2}{*}{$\mathrm{BS}^{\mathrm{a}}$} & \multicolumn{2}{|c|}{$\begin{array}{l}\text { Concor- } \\
\text { dancia }\end{array}$} & \multicolumn{2}{|c|}{$\begin{array}{l}\text { Negativos } \\
\text { falsos }\end{array}$} & \multirow[b]{2}{*}{ BS } & \multicolumn{2}{|c|}{$\begin{array}{l}\text { Concor- } \\
\text { dancia }\end{array}$} & \multicolumn{2}{|c|}{$\begin{array}{l}\text { Negativos } \\
\text { falsos }\end{array}$} & \multirow[b]{2}{*}{ BS } & \multicolumn{2}{|c|}{$\begin{array}{l}\text { Concor- } \\
\text { dancia }\end{array}$} & \multicolumn{2}{|c|}{$\begin{array}{l}\text { Negativos } \\
\text { falsos }\end{array}$} & \multirow[b]{2}{*}{ BS } & \multicolumn{2}{|c|}{$\begin{array}{c}\text { Concordancia } \\
\text { total }\end{array}$} & \multirow{2}{*}{$\begin{array}{c}\begin{array}{c}\text { Negativos } \\
\text { falsos }\end{array} \\
\text { No. }\end{array}$} \\
\hline & & No. & $\%$ & No. & $\%$ & & No. & $\%$ & No. & $\%$ & & No. & $\%$ & No. & $\%$ & & No. & $\%$ & \\
\hline VIH & 40 & 40 & 100 & 0 & 0 & 2 & 2 & 100 & 0 & 0 & 1 & 1 & 100 & 0 & 0 & 43 & 43 & 100 & 0 \\
\hline $\mathrm{HBsAg}$ & 36 & 71 & 98,62 & 1 & 1,38 & 6 & 12 & 100 & 0 & 0 & & & & & & 42 & 83 & 98,8 & 1 \\
\hline Chagas & 42 & 82 & 97,6 & 2 & 2,38 & & & & & & 1 & 2 & 100 & 0 & 0 & 43 & 84 & 97,7 & 2 \\
\hline Sífilis & & & & & & & & & & & 42 & 78 & 92,8 & 6 & 7,2 & 42 & 78 & 92,9 & 6 \\
\hline HTLV & 1 & 1 & 100 & 0 & 0 & & & & & & & & & & & 1 & 1 & 100 & 0 \\
\hline Total & & 271 & 97,84 & 6 & 2,16 & & 27 & 100 & 0 & 0 & & 85 & 93,5 & 6 & 6,5 & & 383 & 96,9 & 12 \\
\hline
\end{tabular}

a BS: Número de bancos de sangre que realizaron la prueba correspondiente.

ciones de los 6 marcadores) y, en algunos casos, prácticamente todas las muestras fueron evaluadas por esta técnica (97\% para la enfermedad de Chagas). La gran mayoría de los sistemas empleados $(60 \%)$ eran de tercera generación.

Las casas comerciales que se utilizaron con mayor frecuencia fueron $\mathrm{Ab}$ bott (Biotech Ltd., Reino Unido), Organon Teknika (Holanda) y Murex (Reino Unido) en el caso de pruebas ELISA, y Organon Teknika y Biobacter (Bogotá, Colombia) en el caso de pruebas de hemaglutinación no treponémicas.

Los bancos de sangre notificaron un total de 1002 resultados negativos concordantes y 49 (5\%) resultados positivos falsos o discordantes, los cuales se distribuyeron en: sífilis, 30,6\%;
HBsAg, 22,5\%; anti-VHC, 20,4\%; antiVIH, 12,2\%; anti-HBc, 8,2 \%; y T. cruzi $6,1 \%$ (cuadro 1 ). Un banco de sangre notificó reacciones positivas a antiVIH en los 6 sueros del panel.

En cuanto a los sueros positivos a los diferentes marcadores infecciosos, se notificaron 383 resultados positivos concordantes y 12 (3\%) resultados negativos falsos, distribuidos de la siguiente forma: sífilis, 50\%; enfermedad de Chagas, 16,7\%; anti-HBc, 16,7\%; HBsAg, $8,3 \%$; anti-VHC, $8,3 \%$ (cuadro 2 ).

Se encontró que, tanto para sueros positivos como negativos, la prueba de ELISA mostró una concordancia de $98,3 \%$, la cual fue significativamente mayor $\left(\chi^{2}=33,46 ; P<0,01\right)$ que la observada con el MEIA (90\%) y la hemaglutinación (89\%).
La mayor proporción de resultados falsos negativos se notificó con la prueba de hemaglutinación (6 de 12), en particular la prueba detectora de la sífilis. De los 49 resultados positivos falsos, $79,59 \%$ (39) se presentaron en 23 de los bancos de sangre que captaban menos de 6000 unidades de sangre por año; $14,28 \%$ (7) se notificaron en cinco de los bancos que captaban de 6000 a 12000 unidades de sangre; y $6,2 \%$ (3) en un banco que captaba más de 12000 unidades (figura 1).

De los 12 resultados negativos falsos, $83 \%$ (10) se presentaron en el grupo de bancos de sangre que captaban menos de 6000 unidades de sangre al año; los dos resultados restantes $(17 \%)$ se presentaron en el grupo que captaba de 6000 a 12000 unidades. No se presen- 
FIGURA 1. Resultados positivos falsos, según las unidades de sangre captadas en los bancos, Colombia, 1998

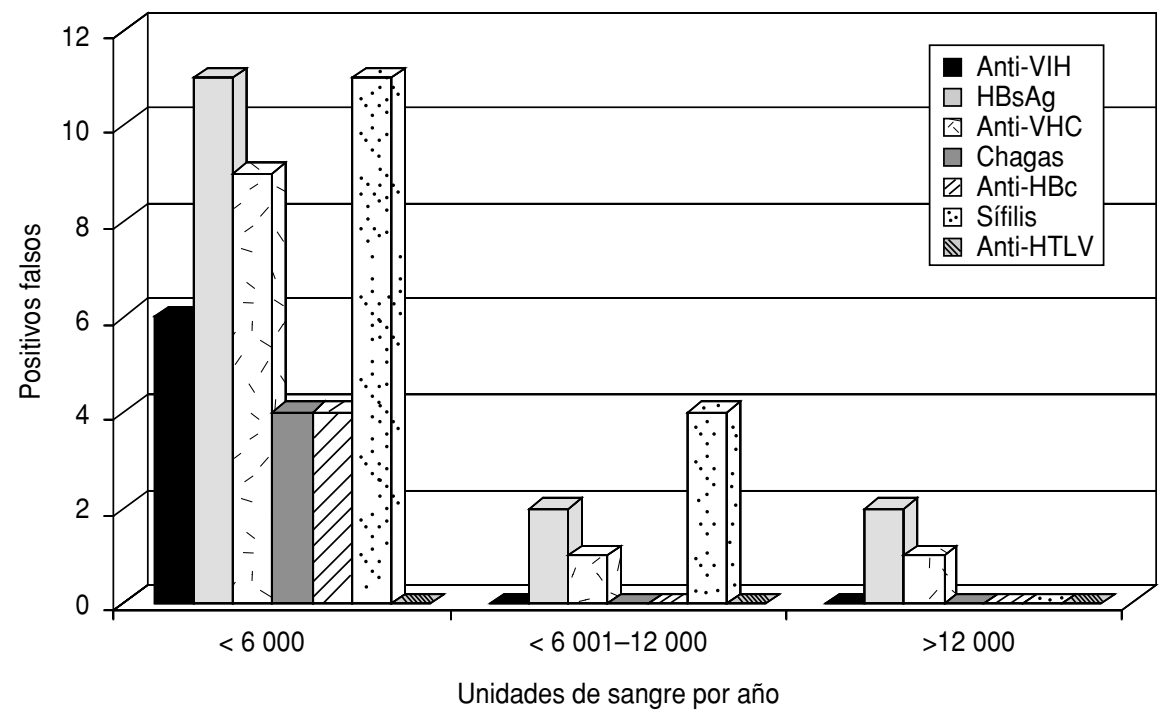

FIGURA 2. Resultados negativos falsos, según las unidades de sangre captadas en los bancos de sangre, Colombia, 1998

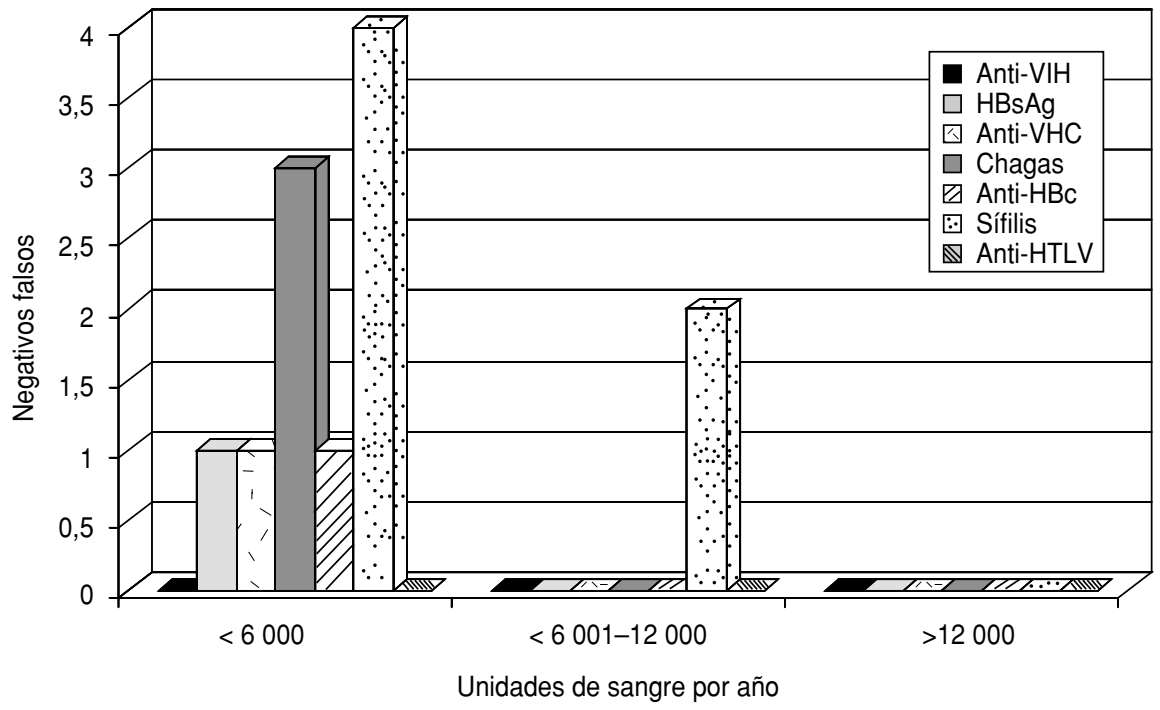

taron resultados negativos falsos en los bancos que captaban más de 12000 unidades de sangre (figura 2).

\section{DISCUSIÓN}

Los resultados del PEC reflejaron deficiencias en los procedimientos de tamizaje de las donaciones en los bancos de sangre. Se obtuvieron 12 (3\%) resultados negativos falsos, lo cual indica que varias personas podrían haber adquirido enfermedades debido a deficiencias en el tamizaje. La presencia de resultados negativos falsos exige una revisión minuciosa de todos los procedimientos realizados, ya que estas pruebas no se repiten y la decisión de declarar una unidad de sangre apta para transfusión se basa en ese único resultado (12).
Cinco por ciento de los resultados positivos falsos podrían deberse a la elevada sensibilidad de las pruebas utilizadas en el tamizaje. Sin embargo, debe observarse, mediante un seguimiento, la tendencia mostrada por este valor, ya que los bancos en Colombia deben repetir estos análisis por duplicado. (Únicamente si dos de las tres pruebas aplicadas a una muestra son reactivas, el resultado se considera positivo y se descarta la sangre). Además, se envía el suero reactivo al laboratorio de referencia para la confirmación del resultado y el asesoramiento del donante (12).

Los PEC tienen un valor muy limitado para determinar la sensibilidad o especificidad de las pruebas debido a que son pocos los sueros y los resultados están sesgados por su selección preliminar. Como el número y el tipo de las muestras escogidas no son representativos del universo que examina cada banco, no es posible emplear los resultados obtenidos para comparar las casas comerciales entre sí. De ahí que en el presente artículo no se discutan los resultados según la casa comercial empleada por los bancos participantes.

La obtención de resultados discordantes con los sueros del PEC no debe ser atribuida exclusivamente a la procedencia y características de las pruebas, sino que debe servir como advertencia para que se revise todo el procedimiento, desde las pipetas, puntas, diluyentes, aparatos en general y los controles internos empleados, hasta la capacitación del personal técnico. Sin embargo, estos resultados del PEC dan una idea del comportamiento de las pruebas de tamizaje sobre el terreno, fuera de las condiciones ideales de una evaluación formal.

Desde 1993, el tamizaje para la detección de anticuerpos contra el VIH, el HBc, el VHC y la sífilis, así como del HBsAg, es obligatorio (13). Sin embargo, en este estudio un banco de sangre no realizó las pruebas detectoras de HBsAg y otro no realizó el tamizaje de la sífilis.

Al seleccionar el tipo de prueba que se va a utilizar en el tamizaje debe tenerse muy en cuenta la necesidad de 
lograr una elevada efectividad en función del costo, y que la prueba tenga la mayor sensibilidad y el mejor valor predictivo positivo posibles. El tipo de prueba utilizada puede variar de un banco a otro debido a factores tales como la disponibilidad de las pruebas comerciales, la infraestructura existente y el conocimiento científico de quien elige las pruebas. No obstante, debe tenerse en cuenta que el tamizaje serológico es un paso fundamental para la obtención de hemoderivados de calidad y para disminuir la transmisión de infecciones por transfusión.

En el caso del banco de sangre que notificó reacciones positivas a antiVIH en todos los sueros, incluidos los negativos, se trata de un grave error. Además de elevar los costos por el desecho de unidades de sangre aptas para transfundir, este tipo de error podría dificultar el asesoramiento adecuado de los donantes positivos. Algunos bancos de sangre no interpretaron correctamente los resultados. Este fallo también debe ser objeto de un cuidadoso análisis, ya que el tamizaje serológico exige tomar una decisión, tanto en relación con la unidad de sangre como en relación con el donante.

La tasa de respuesta de $93 \%$ fue satisfactoria. Sin embargo, si se toma en cuenta que organizar, distribuir y elaborar los paneles para el PEC resulta muy costoso y que para obtener el máximo beneficio de este programa los bancos participantes deben examinar todos los sueros y notificar sus resultados adecuadamente, es necesario elevar aun más esta tasa de respuesta.

El hecho de que ochenta por ciento de los resultados negativos falsos se hayan obtenido en los bancos de sangre que captan menos de 6000 unidades de sangre al año indica que el rendimiento de esos bancos es deficiente, a diferencia del de los bancos de sangre que captan un mayor número de unidades al año. Esto se debe, posiblemente, a que los bancos de sangre de menos complejidad tienen menos práctica en el tamizaje y controlan menos sus procedimientos. Este hecho resalta, una vez más, la necesidad de que en aquellas zonas con bancos de sangre pequeños se refuercen los controles de calidad o, en su lugar, se provean hemoderivados certificados por bancos de sangre de mayor complejidad, los cuales, como demuestra la presente investigación, poseen controles de calidad más estrictos.

El PEC busca, mediante la autoevaluación, que los bancos de sangre conozcan mejor su capacidad en conexión con el tamizaje de marcadores infecciosos. No obstante, esto solo beneficiará a los bancos participantes si los sueros del panel y las muestras sometidas al tamizaje diario se procesan de manera idéntica. Solo así los problemas observados con los sueros del PEC podrán reflejar las dificultades inadvertidas en la labor cotidiana del banco de sangre y estimular a investigar las causas de los resultados incorrectos, corregir las deficiencias detectadas y mejorar así el proceso de tamizaje.

La evaluación externa mediante paneles de suero no sustituye las medidas internas de control de calidad que debe realizar con regularidad cada banco de sangre. No obstante, la obtención de resultados incorrectos con los sueros del PEC podría revelar la falta de medidas internas de control de calidad o su ineficacia.

La necesidad de fortalecer las acciones para mejorar la calidad del trabajo de los bancos de sangre, especialmente las del control de calidad interno, requiere la continuidad de este Programa Nacional de Evaluación Externa.

Agradecimiento. Los autores agradecen a María Mercedes Santacruz, Clara Inés Agudelo y Marlén de Neira, del Laboratorio Nacional de Referencia del INS de Colombia, el procesamiento de los sueros utilizados, y a Ramiro Cruz, de la Organización Panamericana de la Salud, su apoyo al haber facilitado la capacitación para poner en marcha este programa.

\section{REFERENCIAS}

1. Jackson JB, Balfour HH. Practical diagnostic testing for human immunodeficiency virus. Clin Microbiol Rev 1988;1:124-138.

2. Mortier PP. Test for infection with VIH slandered goods. BMJ 1988;296:1615-1616.

3. Yasuhito ABE, Katsuhiko K, Atsushi H, Masazumi M, Shigeru K. Improvement of ELISA sensitivity by allogenic adsorption of polyclonal antibodies: a technical note for nonexperts. Clin Chem Acta 1994;224:103-105.

4. Namu A. Blood transfusion services: organization is integral to safety. Natl Med J India 2001;14(4):237-240.

5. American Association of Blood Banks, Asociación Argentina de Hemoterapia. Manual

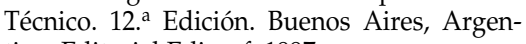
tina: Editorial Edigraf; 1997.

6. Snell JJS, Supran EM, Esparza J, Tamashiro H. World Health Organization quality assess- ment programme on HIV testing. AIDS 1990; 4:803-806

7. World Health Organization. Proposed WHO criteria for interpreting results from western blot assays for HIV-1, HIV-2, and HTLV-I/ HTLV-II. Weekly Epidemiol Rec 1990;65: 281-283.

8. Cortés Buelvas A. ISO 9000 y las buenas prácticas de fabricación de productos sanguíneos. Primera edición. Cali, Colombia: Universidad del Valle; 2001.

9. Rodríguez E, Fernández R, Anerise G, Rainero K, Longo E, Grassi A, et al. Implementación de un sistema de aseguramiento de la calidad en bancos de sangre. Rev Arg Transfusion 2001;27(2):155-165.

10. Hergon E. The quality audit in a blood transfusion center. Transf Clin Biol 1998;5(6): 422-430.
11. International Air Transport Association. Dangerous goods regulators, consolidated version 23. Montreal: IATA; 1982.

12. Cura E, Welder S. Manual de procedimientos de control de calidad para los laboratorios de serología de los bancos de sangre. Washington, D.C.: Organización Panamericana de la Salud; 1994.

13. Ministerio de Salud. Manual de normas técnicas, administrativas y de procedimientos. Capitulo 11. Garantía de calidad. Santa Fe de Bogotá, D.C., Colombia. Imprenta Nacional; 1996.

Manuscrito recibido el 24 de junio de 2002. Aceptado para publicación, tras revisión, el 2 de enero de 2003. 
ABSTRACT Objective. To analyze the serological results found in Colombian blood banks that participate in the external quality program (EQP) of that country's National Institute of Health, in order to improve the quality of the screening of blood for the main serological markers of transfusion-transmitted infectious diseases.

External evaluation of serology results in blood banks in Colombia
Methods. Each blood bank received a panel of six sera with different reactivity and positivity to hepatitis B surface antigen (HBsAg), as well as to antibodies to HIV 1-2, Trypanosoma cruzi (the causative agent of Chagas' disease), Treponema pallidum (the causative agent of syphilis), hepatitis B core ( $\mathrm{HBc}$ ) antigen, hepatitis $\mathrm{C}$ virus (HCV), and human T-lymphotropic virus (HTLV). The screening techniques used were enzymelinked immunosorbent assay (ELISA), microparticle enzyme immunoassay (MEIA), and hemagglutination. With the panel sera, the participating blood banks were asked to apply the same tests that they use on a daily basis to screen blood units and to send their results to the National Blood Banks Unit of the Colombian National Institute of Health.

Results. Of the 46 blood banks participating in the EQP, 43 of them (93\%) returned their results within the requested timeframe. The ELISA test was the one that was used most often $(83.0 \%)$. There were a total of $49(5 \%)$ false positive results and 12 $(3 \%)$ false negative results. Of those 12 false negative results, 6 of them corresponded to the detection of syphilis, 2 to Chagas' disease, 2 to anti-HBc antibodies, 1 to anti$\mathrm{HCV}$ antibodies, and 1 to HBsAg. Eighty percent of the discordant results came from 23 blood banks that each collected fewer than 6000 units of blood per year, and 15\% came from 5 blood banks that collected 6000 to 12000 units per year. One of the blood banks that collected more than 12000 units annually had three false positive results, and none of those larger blood banks had any false positive results.

Conclusions. The percentage of false negative results (3\%) found during the EQP can be considered high, since tests that are negative during blood screening are not repeated, and the decision to declare a unit of blood suitable for transfusion is based on that single result. There is a need to thoroughly review the procedures for screening blood in Colombia, particularly at the centers that performed poorly in this EQP exercise.

\section{Call for Abstracts American Association of Blood Banks Annual Meeting and Transfusion Medicine Expo}

The American Association of Blood Banks (AABB) is seeking abstracts for consideration for presentations to be made at the AABB Annual Meeting and Transfusion Medicine Expo to be held in 1-4 November 2003 in San Diego, California, United States of America. All submissions must be made via an Internet-based "online abstract submitter" on the AABB Web site (http://www.aabb.org). Interested participants do not have to be an $A A B B$ member to propose a meeting presentation.

The deadline for submitting an abstract is 16 May 2003.

Information:

AABB Annual Meeting and TXPO

432 Columbia Street, Unit 11

Cambridge, MA 02141, United States of America

Telephone: (617) 621-1398 • Fax: (617) 621-1423

Email: aabb@dbpub.com

Internet: http://www.aabb.org 